\title{
Fiber Interferometric System for Vehicle Monitoring Near Railway Level Crossings
}

\author{
Stanislav Kepak, Jakub Cubik, David Hruby, Vladimir Vasinek \\ VSB-Technical University of Ostrava, Faculty of Electrical Engineering and Computer Science, \\ Department of Telecommunications \\ 17. listopadu 2172/15, 70800 Ostrava-Poruba, Czech Republic \\ stanislav.kepak@vsb.cz; jakub.cubik@vsb.cz; david.hruby@vsb.cz; vladimir.vasinek@vsb.cz
}

\begin{abstract}
The article describes the development of the vehicle monitoring system that is applicable in the vicinity of the railway level crossings. The proposed system is based on a Mach-Zehnder fiber interferometer sensor, which has a measurement arm embedded in the road surface. The sensor is passive and requires no electrical power at the installation site, immune electromagnetic interference, and is compatible with existing single-mode fiber infrastructure. Experimental measurements were focused on the response of sensors utilizing different mechanical structures of fiber loops, slow-moving vehicles, and sensor function in continuous snow cover. Installed optical fibers in the roadway are regularly monitored and evaluated for their durability. The results have shown that it is possible to detect individual vehicles but above all their axles, which opens the way for vehicle classification. The detection capability of the system is absolute even with continuous snow cover. For slow-moving vehicles, double axle detection occurred due to the longitudinal dimensions of the loop, which will be solved by the second generation of sensors. The resulting system will be installed on a selected railroad crossing as a complement to the existing camera system.
\end{abstract}

Keywords: Fiber optic sensing, Interferometer, Vehicle detection, Vehicle classification, Railway crossings, Obstacle detection.

\section{Introduction}

Transport safety is an essential topic in every country in the world. A large number of collisions, usually with fatal consequences on the property but above all on human lives, are near railroad level crossings. The primary cause of these accidents includes non-observance of road signs or active railroad crossing security; other causes include vehicle defects or faults in the crossing security. These events pose a threat not only to road users but also to persons who travel by train. There are many railway disasters in which the victims were also passengers of the train. Additional protection of railway crossings is thus appropriate the aim of which is to prevent the worst collisions, or at least mitigate the consequences.

Several methods of obstacle detection at level crossings are currently known and used. The most widely used is video or thermal imaging, whether operated manually or automatically [1]. Cameras are installed in a way they can monitor the overall situation at the crossing and near surroundings. The main drawback of the system operating in visible or nearinfrared spectrum is susceptibility to the various lighting conditions and large computational requirements [2]. Other environmental effects such as fog or smoke can present addition difficulty as well. Thermal imaging cameras can overcome some of these limitations; however, there could be an issue with objects with no heat signatures such as unbraked trailers. A combination with other approaches is often advised.

Induction loops are among the oldest systems used. It consists of the coil wire arranged in a loop which is then generating an electromagnetic field. Metallic objects entering the loop area disturbs the field and induces an electrical current which can be analyzed [3]. Loops are usually installed in the road surface in the area before and after the crossing. They can also be installed in the road surface between the tracks; however, this brings further complications (e.g., strong electromagnetic interference). The main drawback is poor detection quality and classification performance of vehicles using aluminum and composite materials, as well as vehicles with high axle-ground clearance.

Modern obstruction detectors are using sound waves, microwaves or light waves. A simple way is to check the socalled "clear path" of a signal between emitter and receiver, where foreign object partially or entirely interrupts the transmission. Advanced systems are using 2D [4] or even 3D [5] images of the level crossing using the static or tilting 
scanners. These systems provide information about the presence of even small objects at the area of interest. Where radio waves based systems have extremely high reliability, their resolution is lower compared to light wave based systems, which are on the contrary susceptible to the water, fog, and dust. Their disadvantage is also the price and the possibility of damage to some of the image sensors (e.g., CMOS) which are unable to withstand the high peak power of light detection and ranging (LIDAR) devices in pulsed mode.

In many countries, including the Czech Republic, there are too many railway level crossings, and it is not economically possible to fit all crossings with expensive technology. The owners of railway infrastructure, often owned by the state, are looking for a balanced technical and economical solution. Many crossings are equipped with CCTV systems capable of monitoring offenses but are not responsible for avoiding accidents. Because of the abovementioned video imaging features, systems should be complemented by another method of event sensing at the railway crossing. One of the possible solutions is to use fiber-optic sensing technologies.

The article describes the vehicle detection system based on fiber interferometer arm embedded in the roadway. The system can be used to detect vehicles in proximity to the level crossing. The principle allows capturing vibrations of moving vehicles passively only using the optical fiber loop installed in the road surface similar to the inductive loop. The advantage is that the optical fiber does not actively emit any electromagnetic field, on the contrary, it is immune to electromagnetic interference. Moreover, they can coexist with existing access and backbone networks which are using the same type of optical fiber [6]. Fiber interferometer can eliminate the general disadvantages of inductive loops. As previously described, fiber optic interferometers are so sensitive that they can monitor the sound and vibration of passing vehicles relatively reliably without having fiber placed in the roadway [7]. However, due to the nature of this application, it is necessary to have absolute detection reliability which can be provided only by the system with embedded fiber in the roadway.

\section{System description}

The proposed sensor system architecture is drawn in Fig. 1. The sensor is powered by narrowband distributed feedback (DFB) laser diode with continuous wave operating at a wavelength of $1550 \mathrm{~nm}$. In the cabinet, placed near the road, passive optical components are located. A single sensor consists of $1 \times 2$ and $3 \times 3$ optical power couplers with an even split ratio which together with two $11 \mathrm{~m}$ long fiber loops are forming balanced Mach-Zehnder fiber-optic interferometer (MZI). The reference arm is located inside the cabinet in the vibration dampening foam, while the measurement arm is pulled out of the cabinet and placed in the narrow $(1 \mathrm{~cm})$ and shallow $(1 \mathrm{~cm})$ loop-shaped groove in the road surface, see Fig. 2a. To the initial experiment, a total of four separately functioning sensors were assembled, and several types of optical fibers differing in the level of protection were placed in the road, see Fig. $2 b$. The groove was then covered with cold asphalt casting compound, see Fig. 2c.

Optical fiber link was used to transmit light to and from the cabinet. The length of the link may be up to several kilometers of single-mode optical fiber. Signal processing unit contains an array of photodetectors (InGaAs photodiodes + electrical amplifiers), the AD converter (National Instruments NI-9220 inside the cDAQ-9181 chassis) and a portable computer. A LabVIEW based application was developed to capture, process, and interpret the sensor signals. Signal processing involves, in particular, the demodulation of the phase difference $\xi(t)$ between the interferometer arms. The three outputs of the $3 \times 3$ coupler produce ideally $120^{\circ}$ mutually phase shifted signals, which can be described by the following equations describing the measurable voltage $u_{i}$ on the three photodetectors.

$$
\begin{aligned}
& \mathrm{u}_{1}= \mathrm{B}_{1}+\mathrm{C}_{1} \cos \left[\xi(\mathrm{t})-\frac{2 \pi}{3}-\delta_{1}(\mathrm{t})\right] \\
& \mathrm{u}_{2}=\mathrm{B}_{2}+\mathrm{C}_{2} \cos [\xi(\mathrm{t})] \\
& \mathrm{u}_{3}=\mathrm{B}_{3}+\mathrm{C}_{3} \cos \left[\xi(\mathrm{t})+\frac{2 \pi}{3}+\delta_{2}(\mathrm{t})\right]
\end{aligned}
$$




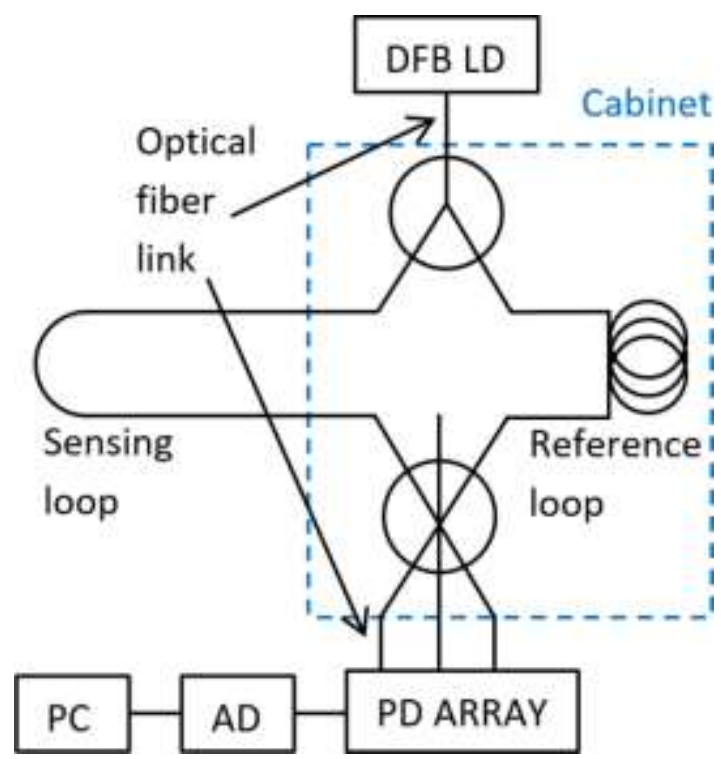

Fig. 1: Vehicle monitoring system architecture.
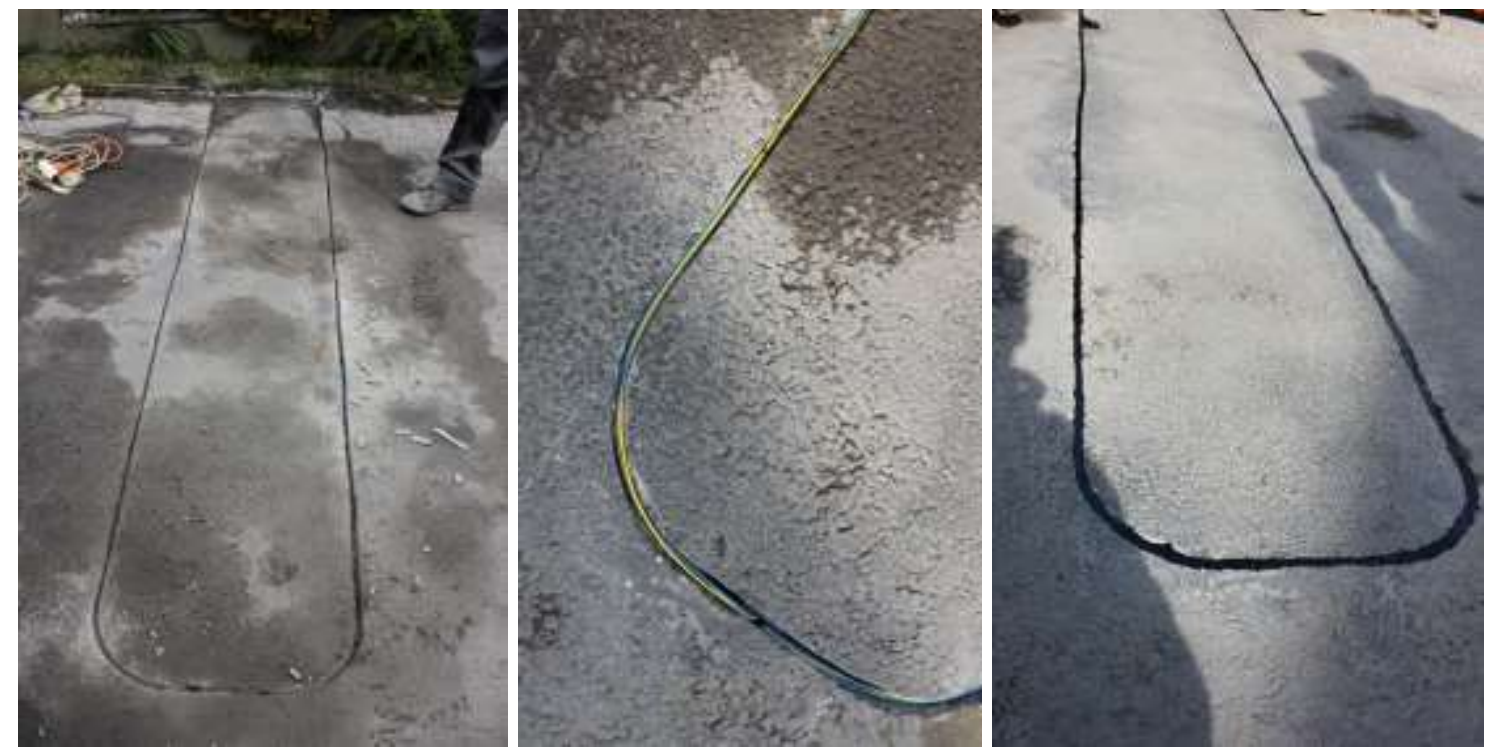

Fig. 2: a) Loop-shaped groove for measurement arms placement. b) Sensing optical fibers placed in the groove.

c) Cold-casted groove with embedded optical fibers.

$\delta_{i}$ is coupler phase asymmetry, $B_{i}$ is a DC offset of cosine modulation, and $C_{i}$ is modulation amplitude. If the DC offset and modulation amplitude is for all three channels the same or compensated the phase difference $\xi(t)$ can be determined using the following formula describing passive homodyne demodulation [8].

$$
\tan \xi(t)=\frac{\sqrt{3}\left(u_{2}-u_{3}\right)}{u_{2}+u_{3}-2 u_{1}}
$$


As the phase term of $\xi(t)$ contains not only a useful signal but also quasi-static drift (temperature, source wavelength changes) it was necessary to suppress this by, for example, high-pass filtration or adaptive filtration [9].

The developed system will be installed in its final form on the selected railroad crossing in that the detectors will always be installed in the pair for one lane (for determining the direction of travel) in front of the crossing and the other pair of sensors behind the level crossing, see the possible fiber installation locations in Fig. 3. The evaluation unit will check whether all the vehicles that have entered the level crossing area have also left it.

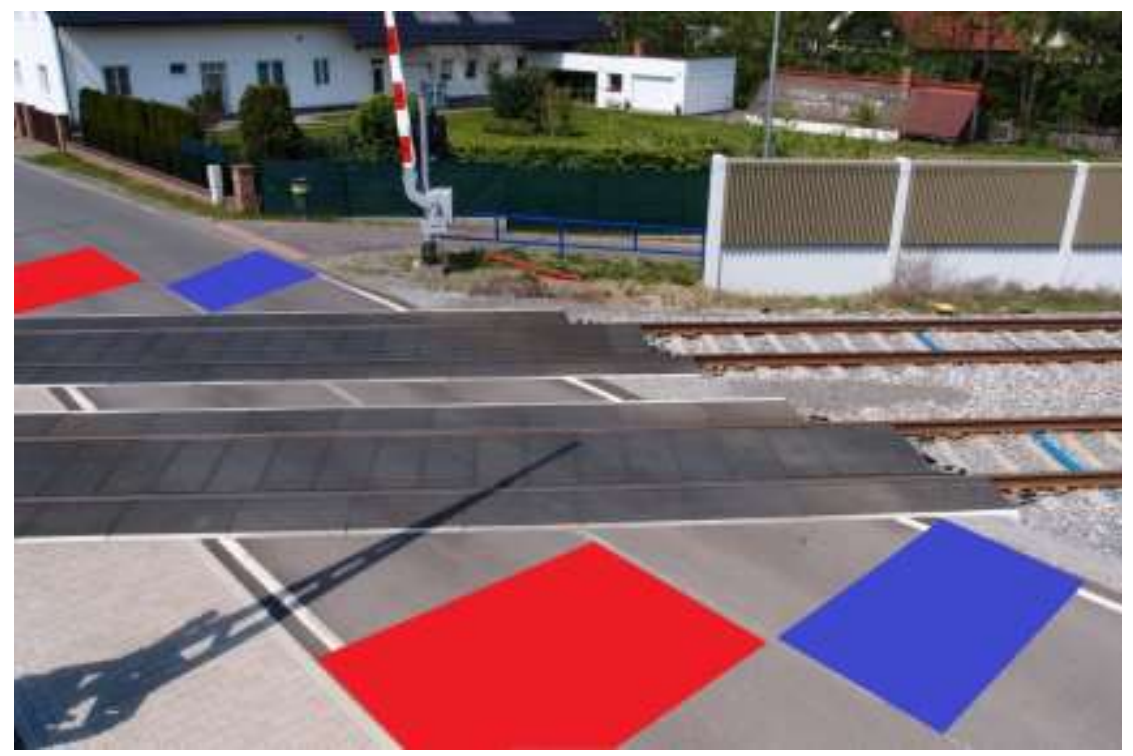

Fig. 3: Possible fiber installation locations in the vicinity of the railway level crossing.

\section{Experimental measurements}

Several experiments with the proposed system have been performed out including attenuation measurements of installed optical fibers with a focus on their durability, comparative measurement of the interferometric responses of individual types of installed fibers, measurement of interferometer responses for continuous snow cover, and finally, experiments aimed at slow drive speed.

Four types of single-mode optical fibers were installed in the road - G.657.A1 fiber in $900 \mu \mathrm{m}$ loose tube protection, fiber in $900 \mu \mathrm{m}$ loose tube with $2.8 \mathrm{~mm}$ outer jacket and aramid yarn tensile protection, $900 \mu \mathrm{m}$ tightbuffered fiber in metal tube with $2.8 \mathrm{~mm}$ jacket and Kevlar tensile protection, and ruggedized fiber optic cable with fiber coated with stainless steel braiding jacket and with outer diameter of $3 \mathrm{~mm}$. The transverse length of the groove was $3.2 \mathrm{~m}$, which allows recording all wheels of each vehicle axle.

The first experiment was focused on detecting the responses of individual sensors to the passage of a passenger vehicle at a constant speed. A sample of the signals from the sensors from one car passage is shown in Fig. 4. It is possible to notice that all the sensors recorded the passage of a vehicle, the two peaks in the signal represent the individual axles of the vehicle, and overall all four responses are very similar in terms of shape and amplitude. It makes the sensor possible to use the sensor as an axle detector. The type of fiber used can be further selected with regard to the required operating life of the sensor or its price. 


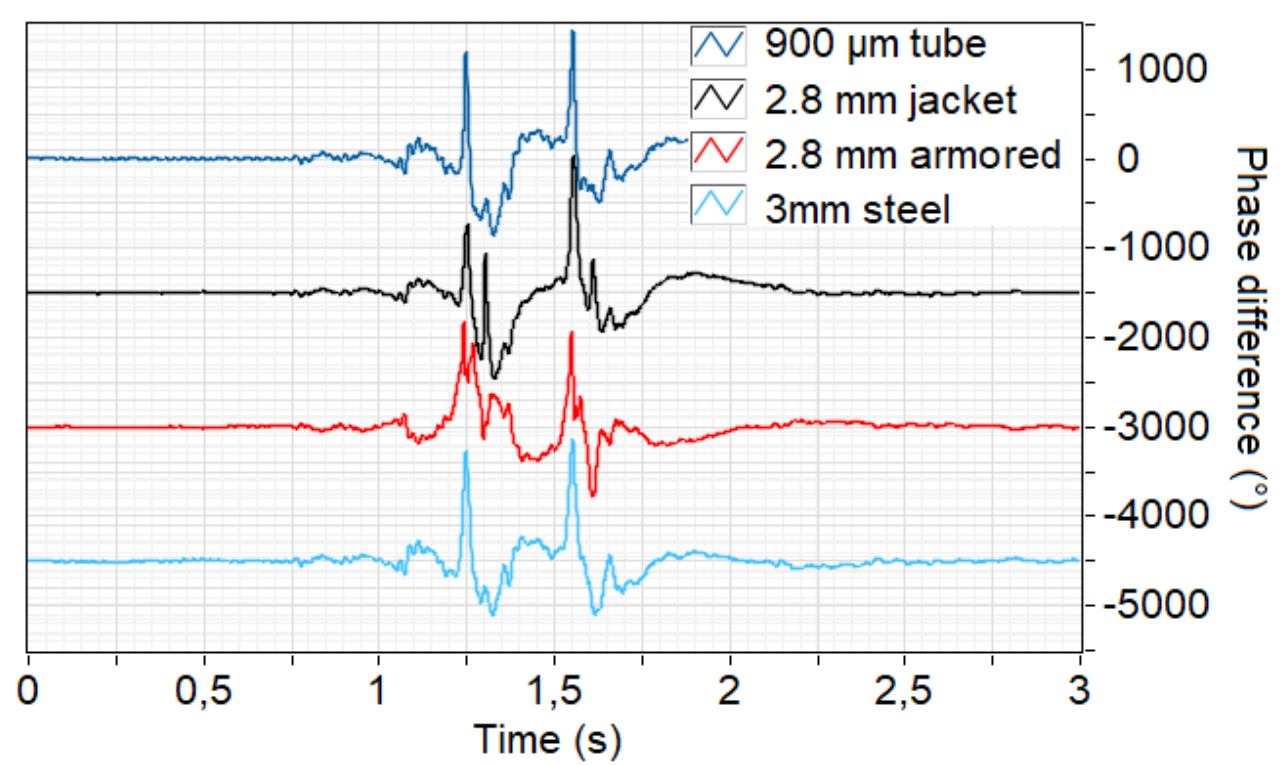

Fig. 4: Phase response of four individual sensors installed in the same groove to a moving vehicle.

Another measurement was focused on the reliability of the system even at low temperatures or when the snow covers the road. All 22 passages made by the test vehicles were recorded by the system, the outside temperature during the measurement was around $-4{ }^{\circ} \mathrm{C}$. For the snow-covered road, the results were the same positive. The measured vehicle speed ranged from 9.3 to $38.4 \mathrm{~km} \cdot \mathrm{h}^{-1}$.

The axle detection algorithm was entirely reliable for vehicles driving at higher speed (approx. $17 \mathrm{~km} \cdot \mathrm{h}^{-1}$ and more), i.e., it detected both axles without false alarms. However, slow-moving vehicles were often evaluated as two vehicles with two axles traveling close behind, see Fig. 5. A wide range of design speeds does not allow introducing the guard interval. The error arises mainly due to the second detection of a single axle as the axle intersects the sensor loop in the road in two relatively distant (the bending radius of the loop is approximately $20 \mathrm{~cm}$ ) points, see Fig. 2.

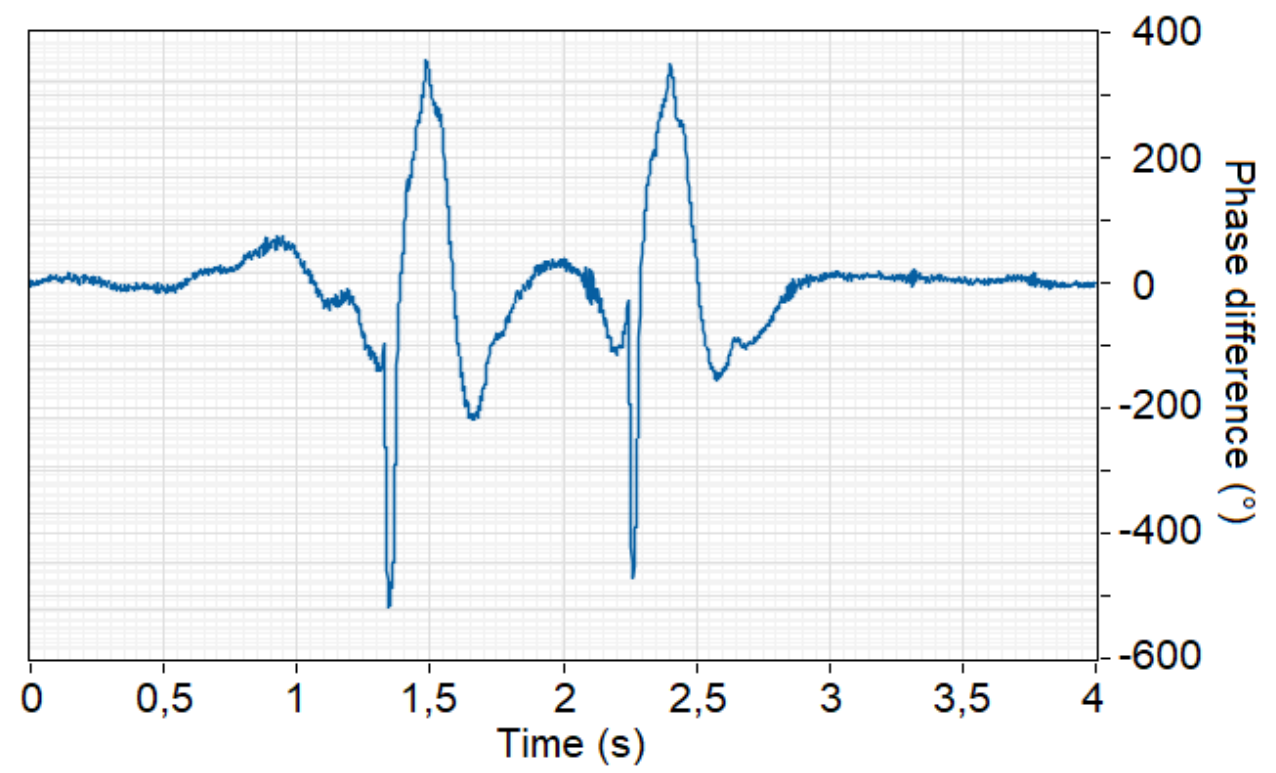

Fig. 5: Phase response to a slow-moving vehicle $\left(11 \mathrm{~km} \cdot \mathrm{h}^{1}\right)$. 
An important aspect is the lifetime of the entire system, so the installed optical fibers were regularly monitored. The observed parameter was fiber attenuation at a working wavelength of $1550 \mathrm{~nm}$. The measurements were made using the insertion loss method described in the standard IEC 60793-1-40:2001 [10], the backscattering method could not be used because of a too short section of the fiber under test. Table 1 shows the average of ten attenuation measurement for each fiber after the production, several days after installation and approximately one year after installation. The results show a minimal attenuation for all installed fibers, small differences in measured values may be due, for example, to the use of different mating sleeves overtime.

Table 1: Average measured attenuation of the measurement arm fibers.

\begin{tabular}{|c|c|c|c|c|}
\hline $\begin{array}{c}\text { Average attenuation } \\
\text { @ 1550nm }\end{array}$ & $900 \mu \mathrm{m}$ tube & $2.8 \mathrm{~mm}$ jacket & $2.8 \mathrm{~mm}$ armored & $3 \mathrm{~mm}$ steel \\
\hline $\begin{array}{c}\text { after production } \\
\begin{array}{c}\text { several days after } \\
\text { installation }\end{array}\end{array}$ & $0.33 \mathrm{~dB}$ & $0.45 \mathrm{~dB}$ & $0.22 \mathrm{~dB}$ & $0.43 \mathrm{~dB}$ \\
\hline $\begin{array}{c}\text { one year after } \\
\text { installation }\end{array}$ & $0.33 \mathrm{~dB}$ & $0.39 \mathrm{~dB}$ & $0.30 \mathrm{~dB}$ & $0.38 \mathrm{~dB}$ \\
\hline
\end{tabular}

\section{Discussion}

The article briefly describes the development of a fiber-optic based vehicle/axle detection system that will be installed in the vicinity of the railway level crossing. The developed system will serve as a supplement to the video imaging obstacle detection system. The combination of these fundamentally different technologies is intended to guarantee minimal error rate and high reliability of the detection system.

The MZI-based system part has the sensing fiber loop embedded in the roadway, similar to the inductive loop detector. It has turned out that, as the loop was made with a large bend radius, this method of installation poses a problem with incorrect detection of the axles of slow-moving vehicles. The solution to this is to install the loop with a small bending radius, close to the critical values of the bending radius of the single-mode optical fiber. Another option is to use a Michelson interferometer, which uses only a section of fiber terminated by a reflective element (e.g., mirror), thereby also reducing installation requirements. Additional experiments with the revised version of the sensors will focus on monitoring of slow-moving or vehicle stopping in the sensor area. Transverse sensitivity of the sensor will also be essential to find out.

Experience has shown that optical fibers can be installed in the cold-casted groove even with a minimum degree of protection; the fibers then lead into the cabinet in protector. In practice, however, we recommend using at least elementary tensile protection because the resulting sensor response is not dependent on the type of protection used as found. In any case, installed optical fibers will be further monitored, and experience of their operating life may be updated.

\section{Acknowledgments}

This paper was supported by the Ministry of Industry and Trade of the Czech Republic project No. FV 10396 System for smart detection and collision conditions signalling for improving of railway security. The research described in this article could also be carried out thanks to the active support of the project No. SP2019/80. This research has been partially supported by the Ministry of Education, Youth and Sports of the Czech Republic through the grant project No. CZ.1.07/2.3.00/20.0217 within the frame of the operation program Education for Competitiveness financed by the European Structural Funds and from the state budget of the Czech Republic. 


\section{References}

[1] H. Salmane, L. Khoudour and Y. Ruichek, "Improving safety of level crossings by detecting hazard situations using video based processing," IEEE International Conference on Intelligent Rail Transportation Proceedings, Beijing, 2013, pp. 179-184.

[2] R. Marik and L. Bohac, "Linear watershed algorithm for 1D real signals," 4th International Conference on Systems and Informatics (ICSAI), Hangzhou, 2017, pp. 1103-1108.

[3] J. Gajda, R. Sroka, M. Stencel, A. Wajda and T. Zeglen, "A vehicle classification based on inductive loop detectors," in Proceedings of the 18th IEEE Instrumentation and Measurement Technology Conference: Rediscovering Measurement in the Age of Informatics, Budapest, 2001, vol. 1, pp. 460-464.

[4] A. Hari Narayanan, P. Brennan, R. Benjamin, N. Mazzino, G. Bochetti and A. Lancia, "Railway level crossing obstruction detection using MIMO radar," 8th European Radar Conference, Manchester, 2011, pp. 57-60.

[5] V. Amaral, F. Marques, A. Lourenço, J. Barata and P. Santana, "Laser-Based Obstacle Detection at Railway Level Crossings," Journal of Sensors, pp.1-11, 2016.

[6] P. Munster, J. Vojtech, T. Horvath, O. Havlis, M. Slapak, P. Skoda, J. Radil, M. Hula, R. Velc, "Coexistence of access and backbone networks with sensor systems," 2016 International Workshop on Fiber Optics in Access Network (FOAN), Lisbon, 2016, pp. 1-5.

[7] J. Nedoma, M. Fajkus, R. Martinek, P. Mec, M. Novak, J. Jargus and V. Vasinek, "Fiber-optic sensor for monitoring a density of road traffic," in Proc. SPIE 10440, Optical Materials and Biomaterials in Security and Defence Systems Technology XIV, 2017.

[8] M. D. Todd, G. A. Johnson and C. C. Chang, "Passive, light intensity-independent interferometric method for fibre Bragg grating interrogation," in Electronics Letters, vol. 35, no. 22, pp. 1970-1971, 1999.

[9] R. Martinek, J. Vanus, M. Kelnar, P. Bilik and J. Zidek, "Application of Recursive Least Square Algorithm to Adaptive Channel Equalization," XXI IMEKO World Congress Measurement in Research and Industry, 2015.

[10] IEC 60793-1-40, "Optical fibres - Part 1-40: Measurement methods and test procedures - Attenuation," 2001 [Online]. Available: https://webstore.iec.ch/publication/3452 